\title{
SIRINGOCELE IMPERFORADO DE LAS GLÁNDULAS DE COWPER. TRATAMIENTO POR VÍA LAPAROSCÓPICA
}

\author{
M. CERQUEIRA, L. XAMBRE, V. SILVA, R. PRISCO, R. SANTO, R. LAGES, M. ALMEIDA, \\ P. PETRACCHI, F. CARREIRA
}

Servicio de Urologia del Hospital Pedro Hispano. Matosinhos. Portugal

Actas Urol Esp. 28 (7): 535-538, 2004

\section{RESUMEN}

SIRINGOCELE IMPERFORADO DE LAS GLÁNDULAS DE COWPER. TRATAMIENTO POR VÍA LAPAROSCÓPICA

OBJETIVO: Los autores presentan el caso clínico de un siringocele imperforado, diagnosticado en un adulto y tratado por cirugía laparoscópica.

MATERIAL Y MÉTODOS: Paciente de 44 años que acude al Servicio de Urgencia por retención urinaria aguda y antecedente de leves síntomas obstructivos del aparato urinario inferior. Presenta al toque rectal tumefacción de la pared rectal izquierda.

El enfermo fue sometido a uretrocistoscopia, ecografia y uretrografía retrógrada. El diagnóstico se obtuvo con Tomografia Computerizada y Resonancia Magnética Nuclear.

RESULTADOS: La marsupialización del siringocele por vía laparoscópica permitió la resolución del cuadro clínico con buena evolución post-operatoria. El paciente tuvo alta hospitalaria a las 48 horas sin necesidad de sonda vesical. Tras seis meses de seguimiento se encuentra asintomático.

CONCLUSIONES: El siringocele es una deformidad rara que habitualmente no provoca sintomas. Puede ser de etiología congénita o adquirida, clasificándose en tres tipos morfológicos: simple, imperforado, perforado o roto. El tratamiento más frecuentemente utilizado es la marsupialización por vía endoscópica dejando una abertura amplia de la cavidad para la uretra. Algunos autores recomiendan su escisión por cirugía abierta, especialmente cuando forma absceso. El abordaje laparoscópico constituye una alternativa mínimamente invasiva con buenos resultados. En los casos asintomáticos diagnosticados incidentalmente se debe optar por una actitud expectante.

PALABRAS CLAVE: Glándulas de Cowper. Siringocele imperforado. Laparoscopia.

\section{ABSTRACT}

IMPERFORATE SYRINGOCELE OF THE COWPER'S GLANDS LAPAROSCOPIC TREATMENT

OBJECTIVES: The authors present a clinical report of an imperforate syringocele diagnosed on a male patient and treated by laparoscopic surgery.

MATERIAL AND METHODS: A forty-four year old patient attends the emergency room with acute urinary retention. With a former history of mild obstructive voiding symptoms, he presented, at rectal examination, a bump on the left rectal wall. This patient also underwent urethrocistoscopy, sonography and retrograde urethrography. To establish the diagnose, Computerized Tomography (CT) scan and MRI were used.

RESULTS: The syringocele marsupialization by laparoscopic surgery, allowed the resolution of the clinical problem, with a good outcome. The patient was discharged 48 hours after surgery, with no need of a vesical catheter. A six month follow-up showed no recurrence.

CONCLUSIONS: The syringocele is a rare deformity which usually is asymptomatic. Of acquired or congenital etiology, it can be classified in four different morphological types: simple, perforate, imperforate and ruptured. The most frequent treatment is the marsupialization of the syringocele by endoscopic surgery, leaving a broad opening of the cavity to the urethra. Some authors recommend an open surgery excision, specially in the presence of an abscess. The laparoscopic approach becomes a minimally invasive alternative with good therapeutically outcome. In the incidentally discovered asymptomatic patients, an expectant approach is the correct approach.

KEY WORDS: Cowper's glands. Imperforate syringocele. Laparoscopy. 
$\mathrm{L}$ as glándulas bulbouretrales son estructuras ectodérmicas originadas en la uretra bulbomembranosa que surgen a partir de la $12^{\mathrm{a}}$ semana de vida intrauterina.

Fueron descritas por primera vez a finales del siglo XVIII. Están localizadas en la espesura del diafragma urogenital lateralmente a la uretra membranosa, y drenan por un conducto de 3-4 $\mathrm{mm}$ que discurre oblicuamente con orificio paramediano en la uretra bulbar ${ }^{1,2}$.

Las glándulas de Cowper parecen estar relacionadas con la defensa inmunitaria del tracto génito-urinario y con la lubrificación de la uretra durante la eyaculación ${ }^{3}$.

La dilatación quística de los conductos de las glándulas de Cowper fue descrita por primera vez en 1953 por Edling. El término siringocele (del griego: syringo-tubo, cele-dilatación) fue atribuido por Maizels, responsables también de su clasificación $^{4}$.

Los siringoceles son patologías poco habituales y generalmente asintomáticas.

\section{CASO CLÍNICO}

Presentamos el caso clínico de un paciente de 44 años que acude al Servicio de Urgencia por retención urinaria aguda y dolor hipogástrico. El sondaje urinario resolvió de inmediato estos síntomas. El enfermo refería sintomas obstructivos e irritativos leves del aparato urinario inferior desde hace tiempo. Al toque rectal la próstata no era palpable, y se detectó una voluminosa tumefacción de la pared rectal izquierda.

La uretrocistoscopia no evidenció alteraciones. En la uretrocistografía retrógrada se observó una disminución del calibre de la uretra membranosa (Fig. 1). La Tomografía Computorizada (TC) reveló una formación quística de 10 x 10 × 8 cm en la pelvis izquierda que comprimía la próstata y el recto.

En la Resonancia Magnética Nuclear (RMN) se observó una gran formación quística rodeada de una cápsula fina y regular con dos componentes que comunican: uno esférico postero-superior de $10 \mathrm{~cm}$, y otro tubuliforme que se alargaba antero-inferiormente, rodeando totalmente la uretra membranosa hasta la raíz del pene, en donde contactaba con las bases de los cuerpos cavernoso y esponjoso. Esta masa desplazaba superior y

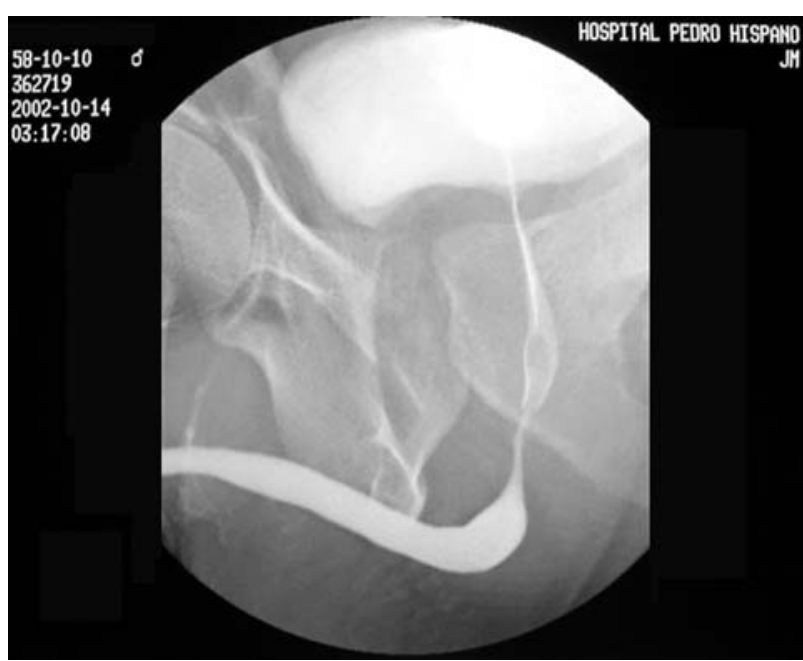

FIGURA 1

lateralmente al recto, próstata, vejiga y vesícula seminal derecha, con claro alargamiento de la uretra membranosa. No se identificó comunicación entre el quiste y las estructuras anatómicas vecinas (Figs. 2 y 3 ).

El paciente fue sometido a drenaje percutáneo perineal de la formación quística, con colación de un dreno perineal. Tuvo alta hospitalaria con micciones espontáneas una vez retirado el dreno.

Un mes después de la intervención realizó una TC de control donde se observó la normal localización de las estructuras pélvicas y la ausencia del quiste, con una pequeña área de contenido líquido rodeando la uretra membranosa.

Una ecografía realizada a los tres meses de la cirugía mostró la recidiva de la formación quística.

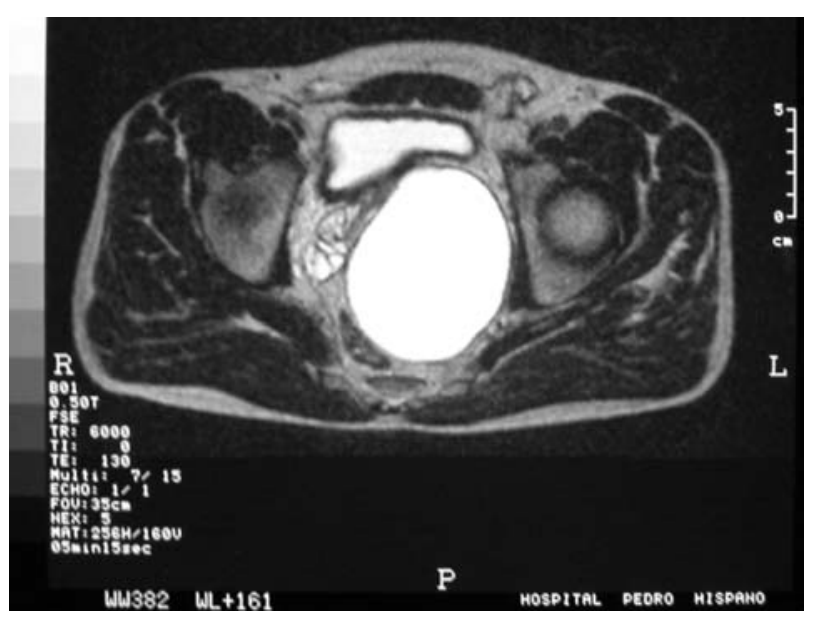

FIGURA 2 


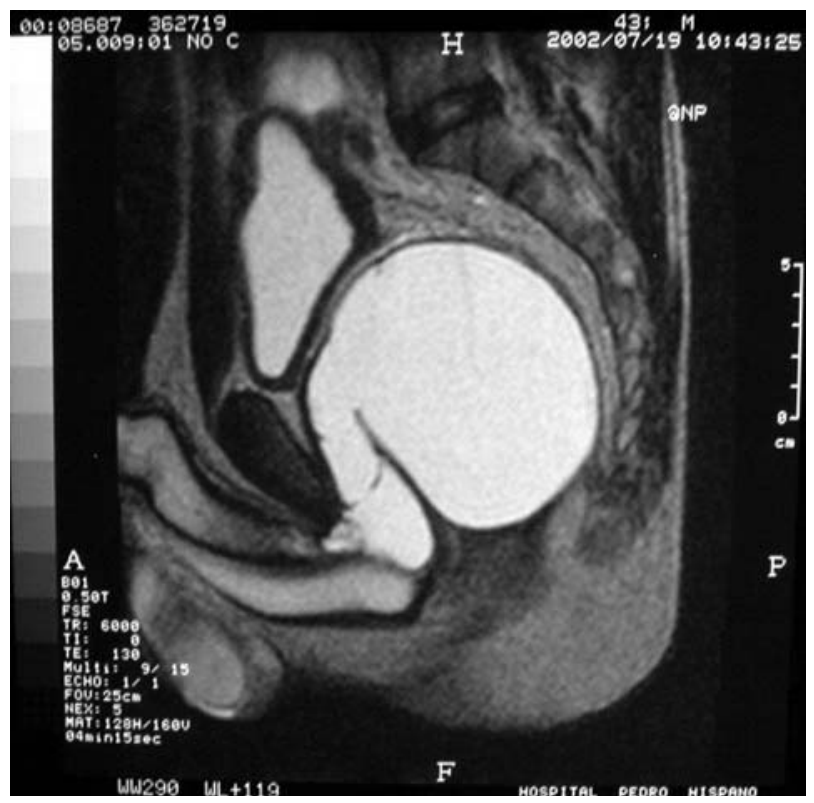

FIGURA 3

Se optó por realizar la marsupialización del quiste para la cavidad peritoneal por vía laparoscópica. El enfermo fue colocado en decúbito dorsal con leve Trendelenburg. Se colocaron tres trocares: uno infraumbilical de $10 \mathrm{~mm}$ colocado según la técnica de Hasson, y otros dos de $5 \mathrm{~mm}$ colocados sobre visión directa en la intersección del borde lateral del recto anterior mayor del abdomen, con una línea imaginaria que une el ombligo con la espina iliaca anterosuperior.

En el fondo de saco de Douglas se identificó una tumefacción quística que colapsaba la vejiga y el recto. Se incidió en su parte superior permitiendo la salida de un líquido incoloro, revertiendo los bordes y dejando una amplia abertura. El interior del quiste presentaba una pared lisa y sin neoformaciones. Para evitar recidivas se procedió a la movilización y sutura de epiplon mayor en el labio posterior de la abertura del quiste (Fig. 4).

El paciente tuvo alta 48 horas después de la intervención quirúrgica, asintomático. En seis meses de seguimiento permanece sin quejas o señales radiológicos de recidiva.

\section{DISCUSIÓN}

Los siringoceles son diagnosticados habitualmente en personas jóvenes, con pocos casos descritos en el adulto. Su incidencia en autopsias es de 2 a 3\% $\%^{5,6}$. En la mayoría de los casos su etiología es congénita, pero también secundaria a procesos infecciosos, inflamatorios o iatrogénicos.

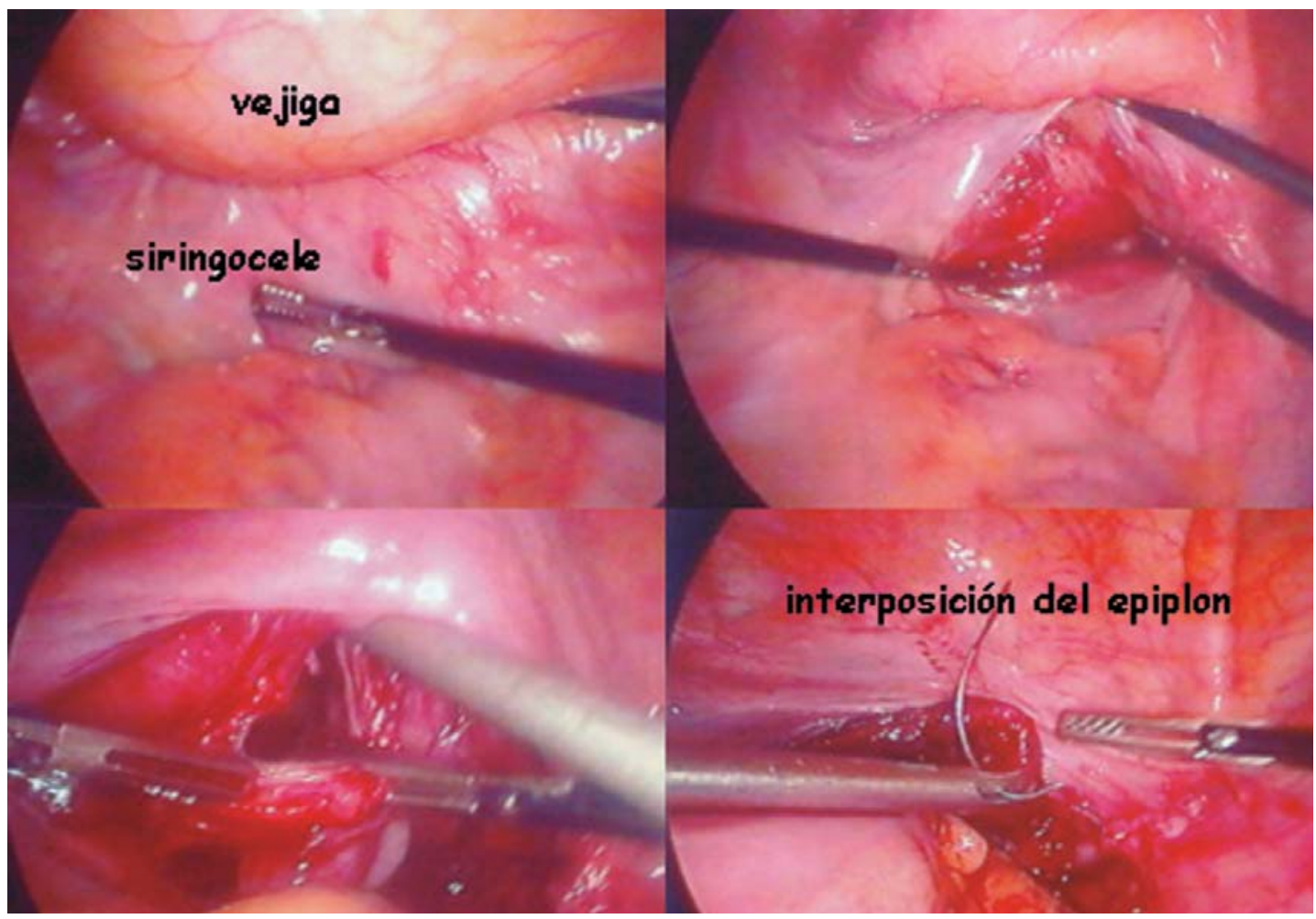


Habitualmente son asintomáticos, aunque pueden causar sintomas irritativos y obstructivos del tracto urinario inferior, hematuria, infecciones recurrentes o goteo terminal en los pacientes jóvenes. La rareza de esta patología y su sintomatología inespecífica lleva a su confusión con otras más vulgares.

Maizels y cols. describen 4 tipos de siringoceles:

- Imperforados: dilatación de la porción distal del conducto glandular. Se traduce radiológicamente como una cavidad quística que comprime estructuras vecinas, afectando más intensamente al tracto urinario inferior.

- Simples: caracterizados por la presencia de una comunicación entre la uretra y el quiste, sin evidencia de lesión traumática. Este orificio ocasionalmente puede ser visible en la endoscopia. La uretrocistografía retrógrada permite comprobar la replección del quiste con contraste.

- Perforado: el siringocele comunica por un gran orificio que no levanta dudas en la endoscopia; la cavidad quística simula un divertículo fusiforme. Estos pacientes frecuentemente refieren pérdidas involuntarias de orina después de la micción.

- Roto: la uretroscopia permite observar la membrana lacerada que separaba la uretra de un siringocele previamente imperforado.

En base a esta clasificación nuestro paciente presenta un siringocele imperforado. Estos siringoceles pueden originarse en la obstrucción del conducto, formando un quiste de retención ${ }^{7}$. La endoscopia puede revelarnos una compresión extrínseca de la uretra, siendo a veces visible una porción de la mucosa más translúcida (que representa el punto común entre la uretra y el quiste). En nuestro paciente no fue posible identificar esta área. La uretrografía retrógrada en estos casos no demuestra la replección del quiste, observándose apenas una imagen de sustracción provocada por la compresión extrínseca del siringocele . $^{2}$

La RMN constituye el medio auxiliar de diagnóstico más reciente en el estudio de estos enfermos. Las imágenes obtenidas nos permiten relacionar las estructuras anatómicas con la formación quística, caracterizarla y ocasionalmente diagnosticarla ${ }^{9}$.
La mayoría de los pacientes no presentan sintomatología que justifique una intervención quirúrgica. La existencia y severidad de los síntomas dictan la necesidad de este tratamiento. El más frecuentemente utilizado es la marsupialización endoscópica del quiste para la uretra, con corte eléctrico o en frío, teniendo la precaución de dejar una abertura amplia para evitar recidivas. Para los siringoceles imperforados algunos autores preconizan la exéresis quirúrgica por vía abierta, con abordaje perineal ${ }^{10}$.

Por la revisión de la literatura no tenemos conocimiento de la utilización de la laparoscopia en el tratamiento de esta patología. Esta vía es una óptima alternativa a las técnicas descritas anteriormente ya que permite el alta hospitalaria en 48 horas después de la cirugía, sin sonda vesical. Los resultados quirúrgicos fueron excelentes, con inmediata resolución de la sintomatología.

\section{REFERENCIAS}

1. CAMPOBASSO P, SCHIEVEN E AND FERNANDES E.: Cowper's syringocele: an analysis of 15 consecutive cases. Arch Dis Child 1996; 75: 71-73.

2. PAUL M et al.: Siringocele de la glándula de Cowper. Arch Esp Urol 2001; 54 (4): 381-383.

3. PASTOR J et al.: Siringocele de la glándula de Cowper. Presentación de dos casos diagnosticados en la edad adulta. Arch Esp Urol 2002; 55 (3): 322-324.

4. PASTOR G et al.: Siringocele simple de la glándula de Cowper. Arch Esp Urol 2001; 54 (2): 163-165.

5. DEWAN P.: A study of the relationship between syringocele and Cobb's collar. Eur Urol 1996; 30: 119124.

6. MERCHANT S, AMONKAR P, PATIL J.: Imperforate syringocele of the bulbourethral duct: appearance on urethrography, sonography, and CT. ARJ 1997; 169: 823-824.

7. BEVERS R, ABBEKERK, BOON T.: Cowper's syringocele: symptoms, classification and treatment of an unappreciated problem. J Urol 2000; 163: 782-784.

8. BRANDES S. RE: Cowper's syringocele: symptoms, classification and treatment of an unappreciated problem. J Urol 2000; 164: 1666-1672.

9. KICKUTH et al.: Cowper's syringocele: diagnosis based on MRI findings. Pediatr Radio 2002; 32: 56-58.

10. RICHTER S, SHALEV M, NISSENKORN.: Late appearance of Cowper's syringocele. J Urol 1998; 160: 128129 .

Dr. M. Cerqueira

Rua Elaine Sanceau, 94 - 2º esq

4465 Sao Mamede de Infesta

(Trabajo recibido el 24 octubre de 2003) 